\title{
Spuren lesen. Evolvierte Voraussetzungen brieflicher Kommunikation
}

\section{Kognitive und Evolutionstheoretische Literaturwissenschaft}

Selbstverständlich ist der Brief ein im historischen Wandel begriffenes Phänomen. In Abhängigkeit von technischen und sozio-kulturellen Faktoren erweisen sich viele Merkmale als variabel, als mehr oder weniger obligatorisch oder randständig. Im Folgenden soll es jedoch nur nebenbei um die kontingente Literatur- und Kulturgeschichte des Mediums und seiner Formate gehen; ich werde den Blick stattdessen auf universale Voraussetzungen und Funktionen brieflicher Kommunikation richten. Gefragt werden soll unter anderem, welche evolvierten, das heißt evolutionär problemlösungsfunktionalen sensomotorischen, kognitiven und emotionalen Adaptionen an brieflicher Kommunikation beteiligt sind. Den methodischen Rahmen für dieses Unterfangen bilden die Kognitive und die Evolutionstheoretische Literaturwissenschaft. ${ }^{1}$ Hierbei handelt es sich um evolutionsbiologisch, kognitions- und neurowissenschaftlich fundierte Literatur- und Medientheorien, die sich für die Wechselwirkung von Natur und Kultur, Biologie und Medien interessieren. Der Ansatz fragt unter anderem, welche universalen, evolutionär adaptiven Anpassungsprogramme, darunter kognitive Dispositionen, an der Entstehung medialer Formen und Inhalte beteiligt sind. ${ }^{2}$ Wie sich zeigen wird, liefert eine solche Herangehensweise auch neue Sichtweisen auf formale und inhaltliche Merkmalsbestimmungen der Gattung Briefroman.

1 Im englischen Sprachraum Literary Darwinisms und Cognitive Poetics. Für deutschsprachige Einführungen in beide Bereiche vgl. Eibl 2010 u. 2016, Mellmann 2006, Wege 2013 u. a.

2 Die Evolutionstheorie erklärt die Entstehung von Adaptionen stets im Hinblick auf ihre Überlebensfunktionalität im Environment of evolutionary adaptedness (EEA). Dabei handelt es sich um jene rekonstruierbare urgeschichtliche Umwelt, in der die Anpassungen entstanden sind. Eine der Kernfragen der Evolutionstheorie lautet, welche Vorteile eine bestimmte Anpassung für das Leben unserer Vorfahren in einer bestimmten Umwelt gehabt haben könnte; vgl. hierzu Tooby u. Cosmides 1992. Paläontologie und Archäologie sind an der Rekonstruktion urgeschichtlicher Umwelten beteiligt, in denen sich Adaptionen für die Gattung Homo als Fitness-steigernd erwiesen haben und langfristig selektiert wurden.

Sophia Wege, Martin-Luther-Universität Halle-Wittenberg

¿ Open Access. ( 2021 Sophia Wege, publiziert von De Gruyter (c))BY-NC-ND Dieses Werk ist lizenziert unter der Creative Commons Attribution-Non-Commercial-NoDerivatives 4.0 International Lizenz. https://doi.org/10.1515/9783110712568-002 
Evolutions- und kognitionstheoretisch argumentierende Literatur-, Medienund Kulturtheorien sind in Großbritannien und in den USA deutlich populärer als in den hiesigen Philologien und stehen längst nicht mehr allein da. Auch in der Paläontologie, Archäologie und Anthropologie gelten evolutionstheoretisch oder neurophysiologisch argumentierende Ansätze mittlerweile als etabliert. So beschränkt sich die Archäologie längst nicht mehr auf die Ausgrabung und Katalogisierung urzeitlicher Artefakte; urzeitliche Spuren wie Steinwerkzeuge, Knochen, Fußabdrücke lassen Rückschlüsse auf Größe, Mobilität und Ernährungsverhalten sozialer Gemeinschaften und somit implizit auch auf den Entwicklungsstand von Kognition und Sprache zu. Aus dem Fortschreiten der Werkzeugtechnik im Verhältnis zur Zunahme des Schädelumfangs schließt die Paläontologie beispielweise, wie sich das Gehirn unserer Vorfahren entwickelte, konkret, wie sie kooperierten und kommunizierten. ${ }^{3}$ Hieraus leitet etwa die Kognitive Archäologie Hypothesen der Co-Evolution von Kultur und Natur/Biologie ab. ${ }^{4}$ Ich greife im Folgenden auf Forschung zurück, die die Evolution des Menschen als rückkoppelnde Verzahnung überlebensdienlicher kultureller und biologischer Faktoren betrachtet. Die Grundannahme lautet, dass Medien immer optimierter auf die evolvierten emotionalen, sensomotorischen und kognitiven Dispositionen der Mediennutzer abgestimmt sind - und aus ihnen hervorgehen. Dies bedeutet, dass evolutionäres Erbe die menschliche Kultur bis heute formt. Kulturmuster, Kommunikationstechniken, Medieninhalte, Gattungen sind nicht nur, aber immer auch, ein Ausdruck unserer Biologie.

\section{Kategorienbildung und Kommunikationsebenen}

Beginnen wir mit einem simplen Experiment und vergleichen wir zwei auf den ersten Blick völlig unterschiedliche Schriftstücke, die unzweifelhaft als ,Briefe“ bezeichnet werden können - ein beliebiges Mahnschreiben aus dem Jahr 2020 und Werthers Brief vom 5. September 1772:

Karl-May-Gesellschaft

Adresse $x x x$

Sophia Wege

Adresse $\mathrm{xxx}$

Mitgliedsnummer

3 Vgl. hierzu Gamble et al. 2014.

4 Vgl. hierzu Mithen 1999. 
MAHNUNG

Sehr geehrte Frau Wege,

der Jahresbeitrag der Karl-May-Gesellschaft e.V. beträgt 39,- Euro. [...] Bitte überweisen Sie den fehlenden Betrag zeitnah, da ansonsten die Auslieferung der Publikation gefährdet ist.

Hierbei unsere Kontodaten: $x x x x x x$

Bitte geben Sie bei der Zahlung die Mitgliedsnummer an.

Mit besten Grüßen,

Ihr XXXXX

Sie hatte ein Zettelchen an ihren Mann aufs Land geschrieben, wo er sich Geschäfte wegen aufhielt. Es fing an: Bester, Liebster, komme, so bald du kannst, ich erwarte dich mit tausend Freuden. Ein Freund, der hereinkam, brachte Nachricht, daß er wegen gewisser Umstände so bald noch nicht zurückkehren würde. Das Billett blieb liegen und fiel mir abends in die Hände. Ich las es und lächelte; sie fragte worüber? - Was die Einbildungskraft für ein göttliches Geschenk ist, rief ich aus, ich konnte mir einen Augenblick vorspiegeln, als wäre es an mich geschrieben. - Sie brach ab, es schien ihr zu mißfallen, und ich schwieg. ${ }^{5}$

Beide Schriftstücke weisen Merkmale auf, die sie uns jenseits aller Unterschiede als Briefe kategorisieren lassen, weil sie unserem mental gespeicherten Prototyp der Gattung mehr oder weniger gut entsprechen. Die Mahnung eröffnet mit einer persönlichen Anrede, allerdings erwarten wir von einem Brief eher einen persönlichen und keinen rein geschäftlichen Inhalt. Werthers Brief weist einen persönlichen Inhalt auf, aber es fehlt die Anrede, die mutmaßlich vom fiktiven Herausgeber weggelassen wurde. Gegenstand seines Briefs an Wilhelm ist wiederum ein Brief Lottes an Albert, der von Werther zunächst als Billett und dann als Zettelchen bezeichnet wird. Da das Schriftstück nicht adressiert ist, darf sich Werther für einen Augenblick einbilden, es sei an ihn gerichtet. Es handelt sich um eine Art Kurznachricht von Lotte an Albert; Zettelchen waren gewissermaßen eine Vorform der Chatnachricht oder SMS. In Abhängigkeit vom zeitlichen Abstand zwischen den einzelnen Kommunikationsakten und vom Intimitätsgrad der Beziehung zwischen den Kommunizierenden werden Chat-Nachrichten mit und ohne Grußformeln versendet, allerdings fehlt ihnen das prototypische Merkmal der Handschriftlichkeit. Schriftstücke, bei denen bestimmte Merkmale wie die Anrede oder persönlicher Inhalt fehlen, bleiben zwar als Briefe identifizierbar, erscheinen dem Rezipienten aber als Beispiele, die vom Prototyp der Gattung abweichen.

Der Brief wird hier mit Verweis auf die kognitionslinguistische Prototypentheorie zunächst grundsätzlich als mentale Repräsentation im Format einer so

5 Goethe 1999 [1778], 169. 
genannten fuzzy category aufgefasst, die eine Reihe unscharfer semantischer Merkmale bündelt. ${ }^{6}$ Den Kern einer Kategorie bildet ein Prototyp, der von Rezipienten als ,bestes Beispiel' wahrgenommen wird. Die Zuordnung von konkreten Einzelexemplaren zu einer Kategorie erfolgt über den Abgleich von Familienähnlichkeiten, das heißt, nicht alle Mitglieder der Familie Brief weisen eine fest definierte Menge von Merkmalen wie beispielsweise Handschriftlichkeit, Adresse, persönlicher Inhalt auf. Vielmehr bestehen zwischen den einzelnen Mitgliedern Übereinstimmungen und Abweichungen hinsichtlich unterschiedlicher Merkmale. Aus diesem Grunde lassen sich sowohl die Mahnung als auch Werthers Brief und Lottes Zettel noch als Briefe kategorisieren, obgleich sie in diversen Merkmalen voneinander und auch vom Prototyp abweichen. Der auch heute noch dominante mental gespeicherte Prototyp der Gattung Briefroman geht auf die populären ,besten Beispiele‘ des achtzehnten Jahrhunderts zurück, weshalb Spielarten des Briefromans im zwanzigsten Jahrhundert nur als Abweichungen wahrgenommen werden.

Allgemein lässt sich festhalten, dass Gattungssystematiken von den Prinzipien unseres kognitiven Kategorisierungsvermögens geprägt sind, was erklärt, dass wir selbstverständlich keine endgültige Definition des Mediums Brief vornehmen können. Das heißt aber nicht, dass man auf Beschreibung und Erklärung kontingenter und universaler Merkmale verzichten müsste. $\mathrm{Zu}$ den Merkmalen des Briefs zählen jene, die die HerausgeberInnen im Exposé zur Marburger Tagung listen, aus der einige Beiträge des vorliegenden Bandes hervorgegangen sind: „Schriftlichkeit, Materialität, Adressiertheit und die Notwendigkeit der Übertragung, Datierbarkeit, Beweglichkeit und zugleich Speicherbarkeit“. Ihre Prototypik zu einem historischen Zeitpunkt $X$ kognitionslinguistisch zu analysieren oder womöglich empirisch zu erheben, bleibt ein Forschungsvorhaben der Zukunft.

\section{Adaptionen im Kontext brieflicher Kommunikation}

Setzt man bei Paul Watzlawicks Kommunikationsmodell ${ }^{7}$ an, umfasst auch die Briefkommunikation grundsätzlich zwei Axiome: Auf der Inhaltsebene dient sie der Übertragung und Speicherung von Informationen, Wissen. Parallel dazu dient jegliche Kommunikation auch der Aushandlung einer Beziehungsebene; dies ist die

6 Rosch u. Lloyd 1978, vgl. hierzu einführend Geeraerts 2006, Kap. 4. Zum Brief vgl. Sinding 2018.

7 Vgl. Watzlawick et al. 1969, Kap. 2. 
Ebene, auf der die von Norbert Bischof fokussierte „Distanzregulation“ stattfindet. ${ }^{8}$ Watzlawick ordnete diesen Ebenen zwei verschiedene Modi der Kommunikation zu: Auf der Informationsebene wird Wissen „digital“ vermittelt; worunter Watzlawick vor allem eine manifeste Propositionalität versteht. Die Beziehungsebene verläuft dagegen analog, das heißt sie wird primär körpersprachlich (akustisch, mimisch, gestisch etc.) kommuniziert. Das kognitionswissenschaftliche Paradigma, unter dem die Körperlichkeit von Kommunikation aktuell geisteswissenschaftlich verhandelt wird, ist embodiment (auch 4 e cognition). ${ }^{9}$ Auf diesen Punkt kann ich im Rahmen des Aufsatzes nicht eingehen; stattdessen soll an dieser Stelle der Beziehungsaspekt stärker in den Fokus rücken, der im Folgenden bindungstheoretisch gefasst wird, und zwar nicht in der psychoanalytischen, sondern in der evolutionsbiologischen Dimension der Bedeutung. ${ }^{10}$ Der Brief ist, oder besser war, ein Medium, das der Aufrechterhaltung überlebensnotwendiger emotionaler Bindungen diente und das zunehmend durch erfolgreichere, digitale Techniken der Bindungsgestaltung wie Chats ersetzt wird. Der Erfolg digitaler Kommunikationstechniken beruht darauf, dass die evolutionär angelegten Bindungsbedürfnisse in räumlicher, zeitlicher und sinnlicher Hinsicht effektiver befriedigt werden. Neben der Bindungsfunktion nimmt ein evolutionstheoretischer Zugang zur Medien- und Gattungsanalyse vor allem die evolvierten kognitiven Dispositionen in den Blick, die an Briefkommunikation beteiligt sind: Das Merkmal Handschriftlichkeit setzt zunächst, und nur scheinbar banal, das körperliche Vermögen voraus, ein Schreibwerkzeug handzuhaben. Der Kommunikationsakt zwischen Sender und Empfänger setzt mündliche Sprachfähigkeit, Lese- und Schreibkompetenz sowie mehrere Teilfunktionen sozialer Intelligenz voraus, darunter das Vorhandensein einer Theorie des Geistes (Theory of Mind), Metarepräsentationsvermögen und Intentionalität. Dieses Inventar der evolvierten Adaptionen, die im Zuge von Briefkommunikation in Dienst genommen werden, wird im Folgenden vorgestellt. ${ }^{11}$

8 Für Details und Differenzierungen vgl. Vellusig 2020, 207-210; vgl. als historische Fallstudie Vellusig 2021.

$9 \mathrm{Zu}$ Theorien der Verkörperung (embodied, extended, enactive and embedded cognition) vgl. beispielsweise den Überblick von Etzelmüller et al. 2017.

10 Vgl. hierzu einführend Reyer 2006.

11 Es handelt sich um eine Auswahl relevanter evolvierter Dispositionen, die ergänzt werden kann. Die genannten Dispositionen spielen selbstverständlich auch bei der Rezeption anderer Gattungen eine Rolle; die Kognitive und die Evolutionstheoretische Literaturwissenschaft sind jedoch bestrebt, die spezifischen Kombinationen von Adaptionen aufzuzeigen, welche an der Rezeption und Produktion der jeweiligen Gattung dominant beteiligt sind, um so eine Art kognitionsästhetisches Profil zu erstellen. 


\subsection{Evolution der Schrift}

Man sollte sich zunächst in Erinnerung rufen, dass der Mensch mehrere Millionen Jahre ohne Briefverkehr, ja ohne Lesen und Schreiben auskam. Die Massenalphabetisierung in den heutigen Industrieländern liegt erst zweihundert Jahre zurück; die Briefkultur des achtzehnten Jahrhunderts war einer Bildungselite vorbehalten. Heute gibt es weltweit fast eine Milliarde Analphabeten; in Asien und Afrika machen sie derzeit etwa die Hälfte der Bevölkerung aus. Es wäre kaum verwunderlich, wenn das in Europa vom Aussterben bedrohte Kommunikationsmedium Brief von den Entwicklungsländern komplett übersprungen würde. Lesen und Schreiben sind menschheitsgeschichtlich betrachtet sehr junge Kulturtechniken, und tatsächlich handelt es sich bei diesen Kompetenzen nicht um überlebensdienliche Adaptionen. ${ }^{12}$

Die Evolution der Schrift erklärt die Kognitive Paläontologie heute wie folgt: ${ }^{13}$ Die ältesten Steinwerkzeuge von Homo erectus, und damit die ältesten Spuren menschlicher Kultur, sind ca. 2,5 Millionen Jahre alt. Neueren Schätzungen zufolge bevölkert der heutige Homo sapiens sapiens die Erde jedoch erst seit circa 200.000 Jahren. $\mathrm{Zu}$ diesem Zeitpunkt war die fundamentale Reorganisation des Gehirns als Adaption an die rauen Umweltbedingungen der Urzeit bereits abgeschlossen. Der an Höhlenmalereien und anderen urzeitlichen künstlerischen Darstellungen festgemachte kulturelle Quantensprung der Menschheit datiert lediglich 40.000 Jahre zurück. Die ältesten Schriften stammen aus dem Jahr 6.000 Jahre vor Christus. Im Zeitraum zwischen 40.000 bis 6.000 vor Christus hat es im Gehirn von Homo sapiens allerdings keine strukturellen Neuerungen mehr gegeben. Die Paläontologie mutmaßt, dass sich in diesem Zeitraum die bereits vorhandenen Intelligenzfunktionen lediglich verstärkten und miteinander vernetzt wurden - man spricht hier von Amplifizierung und gesteigerter kognitiver Fluidität. ${ }^{14}$

Die paläontologische Rekonstruktion der Kulturevolution als Gehirnevolution erklärt, warum es für die Lese- und Schreibfähigkeit im Gehirn keine eigenständige Region gibt: ${ }^{15}$ Lesen und Schreiben wird von mehreren Arealen, vor allem vom Sprachzentrum (Broca-Areal) und den Zentren zur Verarbeitung visueller und sensomotorischer Reize zusätzlich zu anderen Funktionen wie zum Beispiel Raumwahrnehmung und mündlichem Sprachverstehen übernommen. Während es sich also bei dem Vermögen, eine Sprache zu sprechen und zu verstehen, um eine tatsächliche Adaption handelt, gilt die Lese- und Schreibfähigkeit als kulturell

12 Vgl. Varney 2002; Ardila 2014.

13 Vgl. Mithen 1996, 8-13. Vgl. auch Gamble et al. 2014, 72-73.

14 Mithen 1996, 242.

15 Dehaene 2012, 135. 
erworbenes Nebenprodukt der Evolution (byproduct). Beim Lesen und Schreiben werden neuronale Schaltkreise zweitverwertet, die eigentlich für andere Funktionen zuständig sind; man spricht hier von neuronalem Recycling. Die universalen Adaptionen, die Brief- und Chatkommunikation ermöglichen, sind demnach weitaus älter als die Kulturtechnik des Lesens und Schreibens selbst. Allerdings erklären sie, dass die Schriftsysteme aller Kulturen weltweit eine Reihe von Gemeinsamkeiten, Universalien, aufweisen:

Im Falle des Lesens sind kulturelle Invariablen greifbare Realität. Von den chinesischen Schriftzeichen bis hin zum Alphabet beruhen alle Schriftsysteme auf einem morphophonologischen Prinzip: Sie repräsentieren sowohl Wortwurzeln als auch Lautstrukturen. Außerdem stützen sie sich auf einen kleinen Bestand visueller Formen, die überall verwendet werden. Der scheinbaren Vielfalt der Schriften liegt ein weit gespannter Fundus universeller, neurologisch eingeschränkter Merkmale zugrunde. ${ }^{16}$

Zusammenfassend lässt sich feststellen: Briefschreiben und Brieflesen ist mühsam erlerntes, nicht angeborenes Verhalten, das in unserem evolutionären Erbe verankert ist. Menschen schreiben Briefe und tippen Chat-Nachrichten mittels überlebensfunktionaler Dispositionen, die in den vergangenen 200.000 Jahren unverändert geblieben sind. Was sich im Laufe der Kulturgeschichte diversifiziert und optimiert, sind die kulturellen Techniken und Medien, die immer effektiver auf das biologische Erbe abgestimmt sind, die Überlebensprobleme in unserer aktuellen kulturellen Umwelt effektiver bewältigen helfen und unsere evolvierten Bedürfnisse, darunter die Regulierung sozialer Beziehungen (Nähe und Distanz), effektiver zu befriedigen.

\subsection{Werkzeuggebrauch}

Steinwerkzeuge sind die ältesten Zeugen der biologischen und soziokulturellen Evolution des Menschen und als solche von immenser Bedeutung für die Paläontologie. Die ältesten Funde stammen aus der äthiopischen Oldowan-Kultur im Altpaläolithikum vor 2,5 Millionen Jahren. Im Jungpaläolithikum nutzte Homo habilis Faustkeile zur Durchführung diverser überlebenswichtiger Tätigkeiten: der Jagd, der Zerkleinerung von Nahrung, der Bearbeitung von Materialien etc. ${ }^{17}$ Der französische Paläontologe André Leroi-Gourhan vertritt in seinem Klassiker Hand und Wort (1980) die Hypothese von der Co-Evolution von Hand und Gehirn, Werkzeugen und Sprache. Steinwerkzeuge definiert er als exteriorisierte Organe, also

16 Dehaene 2012, 352.

17 Gamble et al. 2014, 72. 

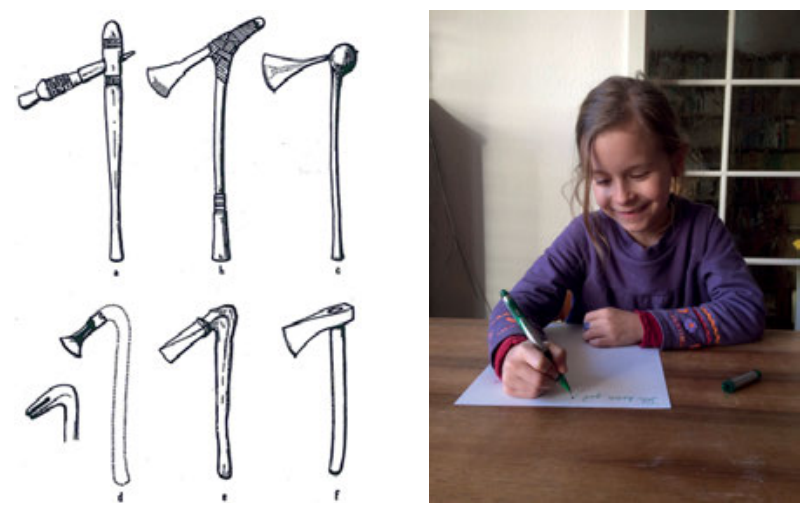

Abb. 1: Urzeitliche Werkzeuge und Gebrauch von Schreibwerkzeug.

Erweiterungen des Körpers und des Gehirns. Bei aller Varianz weisen Faustkeile ebenso wie Schriftsysteme stereotype Formmerkmale auf, die von der Paläontologie als Indizien für das Vorhandensein technischer Intelligenz gewertet werden müssen. Technische Intelligenz gilt wiederum als wesentliche Voraussetzung für die Evolution von Schriftsystemen.

[Der Mensch stellt] konkrete Werkzeuge und Symbole her, die beide auf den gleichen Prozeß, oder besser auf die gleiche Grundausstattung im Gehirn zurückgehen. Dies führt zu der Feststellung, dass die Sprache nicht nur ebenso charakteristisch für den Menschen ist wie das Werkzeug, sondern daß beide der Ausdruck ein und derselben Eigenschaften sind [...]. ${ }^{18}$

Das Schreiben eines prototypischen handschriftlichen Briefs erfordert den adäquaten Gebrauch, d.h. intentionales Greifen, Halten, Bewegen, Führen eines Schreibwerkzeugs mit einer Hand. Der Gebrauch von Feder oder Stift beruht demnach auf der Indienstnahme sensomotorischer Fähigkeiten, die urgeschichtlich der Handhabe von Steinwerkzeugen dienten.

Leroi-Gourhans Thesen konnten mittlerweile auch experimentell belegt werden: In der Oldowan-Kultur wurden Kenntnisse über den Werkzeuggebrauch vorsprachlich mittels Gestik und Imitation weitergegeben. Die Durchsetzung neuer Werkzeugformen im Acheuléen, einer etwas jüngeren afrikanischen Kultur im Altpaläolithikum, erklärt die Paläontologie damit, dass Wissen zunehmend mittels Sprache, und damit sehr viel effektiver, inter- und transgenerationell weitergegeben wurde. Die Durchsetzung neuer Werkzeuggenerationen korreliert zeitlich und räumlich mit einer durch Schädelfunde ermittelten Vergrößerung des Gehirns.

18 Leroi-Gourhan 1980, 149. 
Körperwachstum und Schädelwachstum gelten als belastbare Indikatoren für die Zunahme von Intelligenz. ${ }^{19}$

$\mathrm{Zu}$ den zentralen Befunden der evolutionsbiologisch fundierten Paläontologie zählt heute, dass die Evolution von Werkzeugtechniken nicht ohne „soziale Transmission“ möglich war. ${ }^{20}$ Die Durchsetzung von Werkzeugtechnik und symbolbasierter Kommunikation in immer größer und mobiler werdenden sozialen Gemeinschaften sowie die Steigerung der auf Kooperation basierenden technischen und sozialen Intelligenz gingen vor ca. zwei Millionen Jahren Hand in Hand.

\subsection{Spurenlesen}

Brieflesen ist Spurenlesen. Ein handschriftlicher Brief ist ein Stück Papier, das mit einer Tintenspur versehen ist, die von der Hand eines Schreibers hinterlassen wurde. Im Akt des Lesens wird von der Spur auf einen abwesenden Verursacher und auf einen in der Vergangenheit liegenden Akt der Spurlegung geschlossen. Indem wir die Schriftspur einer Person zuordnen, evozieren wir eine mentale Repräsentation des Schreibenden. Er oder sie scheint durch die Spur und in der Spur anwesend. Die Materialität der Schrift verweist nicht nur, aber auch auf den Körper des Verfassers und lässt sich nicht auf ihre bedeutungstragende Funktion reduzieren. Körperliche, emotionale und kognitive Reaktionen, die durch Zeichen, Briefinhalt und Materialität der Schrift potenziell getriggert werden können, sind psychophysisch real, unabhängig davon, ob es sich um fiktive oder faktische Briefe handelt.

Spurenlesen war eine überlebensdienliche Kompetenz in Jäger- und Sammlergesellschaften:21 Menschen wie Primaten gingen bzw. gehen nicht einzeln, sondern in Gruppen auf Jagd; ein solches Verhalten setzt soziale Intelligenz, Kooperation und Kommunikation unter den Jägern voraus. Zudem muss beim Spurenlesen, anders als bei der Deutung akustischer Reize, von einem visuellen Zeichen auf ein aktuell abwesendes Tier geschlossen werden bzw. auf die Anwesenheit dieses Tiers zu einem früheren Zeitpunkt. Diese höhere Form der imaginativen Inferenzbildung auf Basis visueller Reize erweiterte den Jagdradius, steigerte den Jagderfolg und somit die Überlebensfähigkeit. Aus den genannten Gründen gilt Spurenlesen in der Kognitiven Archäologie als Indikator höherer Intelligenz: „It is likely that humans are the only primate able to draw inferences form visual clues which are displaced

19 Morgan et al. 2015.

20 Gamble et al. 2014, 124-125.

21 Mithen 1996, 183-185. 
from their referent, e.g. footprints".22 Mithen argumentiert, dass Spurenlesen, definiert als die Fähigkeit, unbelebten Objekten eine Bedeutung zuzuweisen, eine der wesentlichen Voraussetzungen für die Evolution von „,natural history intelligence“ ist, und somit als Vorstufe von sozialer Intelligenz zu gelten hat, mittels derer intentionale symbolische Kommunikation möglich wurde:

The capacity to attribute meaning to the unintentionally made tracks and trails of potential prey is a critical development of natural history intelligence. [...] The ability to draw inference from marks such as footprints most likely reaches back to when earliest Homo [...] began hunting and scavenging on the African savannah. ${ }^{23}$

Nichtintentional hinterlassene Tierspuren und intentionale symbolische Schriftspuren weisen viele gemeinsame Merkmale auf, so Mithen: Sie sind unbelebt, räumlich und zeitlich vom Referenten und dem ursächlichen Ereignis der Spurentstehung getrennt, und sie machen die Zuordnung zusätzlicher Bedeutungen erforderlich, welche die Spur selbst nicht direkt abbilden. So bildet zwar ein Hufabdruck den Huf eines Pferdes ab, doch Größe, Gangart oder Laufrichtung müssen geschlussfolgert werden. Spurenlesen auf der Jagd gilt deshalb in der Paläontologie als eine jener Adaptionen, die das Gehirn entscheidend auf das Lesen und somit auch auf Briefkommunikation vorbereitete. ${ }^{24}$

\subsection{Bindungsverhalten}

Phylogenetisch wie ontogenetisch wird Bindungsverhalten zunächst vorsprachlich realisiert, d.h. durch soziales Lächeln und intensiven körperlichen Kontakt. ${ }^{25}$ Das soziale Lächeln gilt als wichtigstes Kommunikationsmittel zwischen Säugling und Bezugsperson. Durch dieses Verhalten werden im Gehirn Hormone freigesetzt, die Vergnügen, emotionale Nähe und somit die überlebensnotwendige Fürsorge der Eltern auslöst. Auch ein Kulturprodukt wie eine Puppe kann evolviertes Fürsorgeverhalten triggern. Ebenso kann ein (fiktiver) Brief evolutionspsychologische Anpassungsprogramme, darunter Bindungsverhalten, in Gang setzen.

Die evolutionstheoretische Erklärung für Bindung und Bindungsverhalten ist Sozialität: „The great evolutionary invention of the primate family is sociality.“26 Ein isolierter Homo sapiens existiert nicht, weil er nicht überlebensfähig wäre; der

22 Mithen 1996, 274.

23 Mithen 1996, 183.

24 Mithen 1996, 184.

25 Gamble et al. 2014, 56.

26 Gamble 2014, 39. 

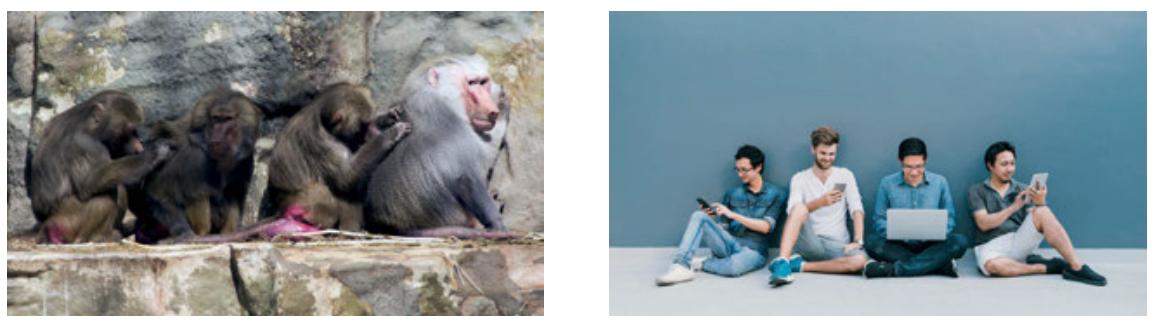

Abb. 2: Kraulende Affen und kraulende Kommunikation.

Mensch lebt in Gruppen, weil sein Überleben, und das heißt grundsätzlich seine Gehirnentwicklung, von emotionalen Bindungen, von Kommunikation und Kooperation abhängt. In urzeitlichen Umwelten bot emotionsbasierte Gruppenkohäsion allen Mitgliedern einer Gemeinschaft Schutz vor Gefahren wie Stammesrivalitäten und den Müttern und Kindern Schutz vor Raubtieren. Bindungsverhalten senkt das Stresslevel im Gehirn, reguliert emotionale Nähe und Distanz, fördert die Fortpflanzung und somit den Fortbestand der Gattung.

Kommunikation dient keineswegs primär dem pragmatischen Austausch von Informationen oder der Wissensspeicherung. Statistisch gesehen hat der Austausch sozialer Informationen, sprich Klatsch und Tratsch, den größeren Anteil an mündlichem Austausch. ${ }^{27}$ Robin Dunbars berühmte Grooming-Hypothese geht auf die Beobachtung zurück, dass Primaten circa 20 Prozent ihrer Zeit damit verbringen, sich gegenseitig das Fell zu entlausen. ${ }^{28}$ Gegenseitige Berührungen setzen Endorphine frei, die angenehme Gefühle auslösen, welche wiederum zu altruistischem Verhalten, beispielsweise auf der Jagd oder bei der Verteilung von Ressourcen, motivieren. Bindungsverhalten stärkt also den sozialen Zusammenhalt unter Primaten auch außerhalb des eigenen Genpools.

Der bindungsabhängige Altruismus korreliert dabei exakt mit der Zeit, die Primaten mit dem jeweiligen Grooming-Partner verbringen. Je öfter und länger der Partner entlaust wird, desto größer ist die Bereitschaft, sich altruistisch zu verhalten. Das Dilemma dieses sozialen Kapitals ergibt sich allerdings aus der Expansion der Gruppengröße, bei Primaten wie beim Menschen. Je größer die sozialen Gemeinschaften, desto mehr Zeit muss in die körperliche Bindungspflege investiert werden und desto weniger Zeit bleibt für andere überlebenswichtige Tätigkeiten wie Nahrungsbeschaffung. Wären Menschen, wie Primaten, allein auf Kraulen angewiesen, um soziale Bindungen aufrechtzuerhalten, müssten wir vier-

27 Dunbar 1998, 13.

28 Dunbar 1998, 102-103. 
zig Prozent unserer Zeit damit zubringen. ${ }^{29}$ Laut Dunbar (1998) ist es die Evolution der Sprache, Klatsch und Tratsch, die dieses Zeitproblem gelöst hat. Sprache löste Kraulen ab und übernimmt überwiegend dieselbe bindungsstärkende Funktion. Sprachliche Kommunikation leistet die Stimulation emotionaler Bindung auch über körperliche, zeitliche und räumliche Distanz hinweg. Dieser Zusammenhang scheint auch auf Brief- und Chatkommunikation übertragbar, anders gesagt, wenn wir einen persönlichen Brief oder eine Chatnachricht schreiben, ,kraulen' wir den Empfänger. Vermutlich ist Bindungsaffirmation auch der Sinn der Grußformeln, denn der informationelle Gehalt von „Herzliche Grüße“ tendiert gegen Null und hat allenfalls Gestaltabschlussfunktion.

In diesem Zusammenhang relevant ist auch der gründlich erforschte Faktor Gruppengröße, d.h. die Anzahl der Personen, mit der eine Einzelperson durchschnittlich in Kontakt steht. Bei aller individuellen Varianz gelten die Zahlen seit der Jäger- und Sammlerzeit als erstaunlich stabil: Wir haben im Schnitt fünf sehr enge, krisenfeste Bezugspersonen, 15 Personen im erweiterten Freundeskreis, 35 im Bekanntenkreis und wir kennen insgesamt ca. 150 Personen persönlich - 150 ist die magische Zahl der Paläontologie (Dunbar's Number). Egal, wie groß die Stadt ist, in der wir heute leben, der soziale Zirkel, in dem sich der Mensch seit der Urzeit bewegt, besteht im Schnitt aus nur 150 Personen..$^{30}$

Tab. 1: Gamble et al. 2014, 42.

\begin{tabular}{lr}
\hline Social groupings among hunter-gatherers & Numbers \\
\hline Tribes (language) & 1500 \\
Mega-Bands (marriage and trade) & 500 \\
Communities / Dunbar's Number & 150 \\
Bands (overnight camps) & 50 \\
Foraging group / support group & 15 \\
Intimiate group / soul mates & 5 \\
\hline
\end{tabular}

Die Zahlen resultieren aus der Größe des Neokortex, dessen Kapazitäten die Zeit begrenzt, die uns im Leben neben anderen überlebenswichtigen Aktivitäten für Bindungspflege zur Verfügung steht. ${ }^{31}$ Die Fähigkeiten des Gehirns sind begrenzt und diktieren damit auch die Zeiträume, die wir für überlebensdienliche Tätigkei-

29 Dunbar 1998, 102.

30 Gamble et al. 2014, 42.

31 Die Angaben beinhalten jeweils alle niedrigeren Levels, d.h. in den 150 sind die fünf engsten Freunde und alle weiteren mit eingerechnet. 
ten brauchen. Zeit ist eine überlebensrelevante Ressource; deshalb setzen sich Medien durch, die an einer Stelle Lebenszeit einsparen, die dann an vorteilhafterer Stelle ökonomischer investiert werden kann. Die qualitativen und quantitativen Vorteile von Chatprogrammen zur Bindungsregulation gegenüber dem Brief liegen auf der Hand. Nostalgiker, die die Körperlichkeit von Handschrift vermissen, scheinen die Tatsache zu verdrängen, dass dieses Defizit beim Chatten durch gesteigerte visuelle, akustische und haptische Befriedigung und durch Zeitersparnis kompensiert wird. Früher warteten Verliebte auf den Briefträger, heute auf den Vibrationsalarm. Die Handys der neuen Generation haben Briefumschlaggröße erreicht. Dass wir in Zeiten größtmöglicher Mobilität zu jeder Zeit, instantan und über immer größer werdende Distanzen hinweg, mittels Chat Spuren lesen und Bindungen aufrechterhalten können, ist auch ein Effekt unseres evolutionären Erbes.

\subsection{Soziale Intelligenz. Theorie des Geistes, Metarepräsentation, Intentionalität}

Theorie des Geistes (Theory of Mind, auch mentalizing und Metarepräsentationsbildung) meint im Kern die Fähigkeit, sich in die Vorstellungswelt anderer hineinzuversetzen, das heißt mental zu modellieren, was ein Gegenüber denkt und fühlt, mentale Repräsentationen von den mentalen Repräsentationen, Zuständen, Überzeugungen und Intentionen der Kommunikationspartner $\mathrm{zu}$ formen. ${ }^{32} \mathrm{Me}$ tarepräsentationsvermögen erlaubt höherstufige Aussagen wie ,ich weiß, dass du weißt, dass ich weiß, dass etc.“. Phylogenetisch entwickelt sich die Theory of Mind zwischen dem dritten und vierten Lebensjahr, erkennbar an der Fähigkeit zu lügen, das heißt die eigene von der fremden mentalen Repräsentation zu unterscheiden und fremde Vorstellungen absichtsvoll zu manipulieren. Ontogenetisch markiert die Theory of Mind einen Meilenstein der kognitiven Evolution, der als Voraussetzung empathischer Interaktion, Kommunikation und somit von sozialem Bindungsverhalten gilt:

We argue that a subconscious, reflexive appreciation of others' intentions, emotions, and perspectives lies at the root of the most complex forms of T[heory] o[f] M[ind] and that these abilities first evolved because natural selection favored individuals that were motivated to attend to other individuals' social interactions and empathize with them. [...] We therefore

32 Vgl. beispielsweise Theory of Mind im Rahmen der Paläontologie: Gamble 2014 et al., 51-52, auch 181-182. 
propose that the evolution of a ToM ultimately derives from its role in facilitating the formation of social bonds. ${ }^{33}$

Das Kommunikationsmedium Brief nimmt die Theory-of-Mind-Fähigkeit des Menschen in besonderem Maße in Dienst. Briefe laden ihre Empfänger ein, sich eine Vorstellung von den Gedanken und Gefühlen der Sender zu machen. Wir nehmen die brieflich vermittelten Inhalte stets als eine vom Verfasser intendierte, aus der Vorstellungswelt des Verfassers resultierende und für den Empfänger präparierte Gabe wahr. Sender und Empfänger unterhalten im Akt der Kommunikation eine mentale Repräsentation des jeweils anderen; das heißt, dass ein Briefschreiber eine hypothetische Vorstellung davon unterhält, welche Vorstellung von ihm und seinen Absichten der Briefpartner vermutlich hat. Beide Briefpartner zielen auf die Veränderung der Vorstellungswelt des jeweils anderen ab und wissen zugleich darum, dass auch der andere sich dessen bewusst ist. Ein kommunikativer Akt beschränkt sich nicht auf den Austausch von Propositionen, sondern stellt auch einen Versuch dar, durch deren Übermittlung die mentalen Repräsentationen des Kommunikationspartners in einem ganz bestimmten Sinne zu manipulieren.

Das macht wohl auch verständlich, weshalb der Briefroman im achtzehnten Jahrhundert zum Faszinosum werden konnten. Der Reiz dieser genuin literarischen Gattung bestand darin, dass er seine Leserinnen und Lesern dazu verführte, die im realen Briefverkehr erprobte Theory of Mind in einer fingierten Kommunikationssituation lustvoll zu reaktivieren. Der Leser ist eingeladen, sich ein mentales Modell der mentalen Repräsentationen der Figuren zu konstruieren bzw. deren Theory-of-Mind-Aktivitäten nachzuvollziehen. Der Leser versteht nicht nur, was die Figuren schreiben, er wird auch angeleitet, darüber nachzudenken und zu schlussfolgern, welche intentionalen Vorstellungen und Gefühle sich ,hinter' den manifesten Informationen verbergen, vielfach auch solche, die von der kommunizierten Semantik abweichen, etwa weil sie dem Briefpartner bewusst vorenthalten werden sollen. Der Briefroman erzeugt somit beim Leser eine Reihe partizipatorischer, ja detektivischer Mitfühl- und Denk-Effekte, etwa die Aufdeckung von Absichten oder Lügen seitens der Figuren. Das Lesen des Briefromans gerät so zum Theory-of-Mind-Training für die Leserinnen und Leser.

Zum Auftakt von Richardsons Pamela (1740) gestaltet sich dieser Mechanismus wie folgt. Aus Pamelas Briefen an die Eltern kann ein Leser mittels Theory of Mind zu einem sehr frühen Zeitpunkt - nämlich viel früher als Pamela selbst - schließen, dass Mr. B. dem jungen Mädchen nicht aus Nächstenliebe eine Anstellung als Haushaltshilfe gibt, sondern dass er sexuelle Absichten verfolgt. Eine Reihe von

33 Seyfarth u. Cheney 2013, 10350. 
Indizien weist den Leser darauf hin, dass von Mr. B. eine Gefahr ausgeht, die die Hauptfigur in ihrer jugendlichen Naivität zunächst nicht erkennt. Auf Grundlage des Textes konstruiert der Leser die fiktiven mentalen Repräsentationen in den Köpfen der verschiedenen Figuren, und zwar als Bestandteile seines eigenen mentalen Modells dieser Figuren, und gleicht diese gegeneinander ab. Sehr bald weicht die mentale Repräsentation von Mr. B’s Absichten im mentalen Modell des Lesers an entscheidenden Punkten von Pamelas mentalen Repräsentationen von Mr. B's Absichten ab. Wenn Richardson im weiteren Verlauf der Briefkommunikation Mr. B's wahre Absichten zu Tage treten, darf sich der Leser in seinen eigenen Vermutungen bestätigt sehen, zu denen er vorher mittels Theory-of-Mind-Kompetenz gelangt war. Der Leser atmet regelrecht auf, als Pamela aus Mr. B’s Briefen endlich die gleichen, richtigen Schlussfolgerungen zieht, wenn sie also die Spuren seiner Briefe ebenso deutet, wie der Leser selbst, so dass die Theory-of-Mind-basierten mentalen Modelle von Leser und Hauptfigur zur Deckung kommen. Zugleich löst die zwischenzeitliche kognitive Diskrepanz zur Figur auf Seiten der Leser Empathie für die blutjunge schutzbedürftige Hauptfigur aus. Der Briefroman führt zu einer verstärkten Stimulation emotionaler und rationaler Dimensionen der Theory-ofMind-Kapazität der Leserinnen und Leser. In dieser Form der Indienstnahme von Bindungsverhalten und Theory of Mind in erzieherischer Absicht kann man ein distinktives Merkmal der kognitiven Poetik der Gattung Briefroman in der Aufklärung sehen.

\section{Fazit}

In Thinking Big. How the Evolution of Social Life Shaped the Human Mind schreiben Gamble, Gowlett und Dunbar, was die Gattung Mensch auszeichne, sei die Fähigkeit, auch über räumliche und zeitliche Distanzen hinweg soziale Kontakte aufrecht zu erhalten: „To live apart, yet stay in touch.“"34 In Pandemie-Zeiten zeigt sich einmal mehr, dass dieser Fähigkeit ein grundlegendes, evolutionär funktionales Bedürfnis zugrunde liegt, das heute durch Chat-Kommunikation befriedigt wird und wohl vor wenigen Jahrzehnten noch durch das Medium Brief gestillt worden wäre. In der englischen Wendung ,stay in touch“ bleibt die physiologische Wurzel dieses Bedürfnisses, der Drang nach körperlicher Berührung, präsent. Das Medium Brief war ein Medium der Berührung, das nun von berührungsintensiveren Medien ersetzt wird.

34 Gamble et al. 2014, 78. 


\section{Literatur}

Ardila, Alfredo (2014). „There is not any specific area for writing: From cave-paintings to computers“, in: International Journal of Psychology, 39: 61-67.

Dehaene, Stanislas (2012). Lesen. Die größte Erfindung der Menschheit und was dabei in unseren Köpfen passiert. München.

Dunbar, Robin (2000). Klatsch und Tratsch. Wie der Mensch zur Sprache fand. München.

Eibl, Karl (2016). Evolution - Kognition - Dichtung. Zur Anthropologie der Literatur. Münster.

Eibl, Karl (2010). „Literaturwissenschaft“, in: Evolution. Ein interdisziplinäres Handbuch. Hg. v. Philipp Sarasin u. Marianne Sommer. Stuttgart u. Weimar: 257-266.

Etzelmüller, Gregor, Thomas Fuchs u. Christian Tewes (2017). Verkörperung. Eine neue interdisziplinäre Anthropologie. Berlin u. Boston.

Gamble, Clive, John Gowlett u. Robin Dunbar (2014). Thinking Big. How the Evolution of Social Life Shaped the Human Mind. London.

Geeraerts, Dirk (2006). „Prototype Theory. Prospects and Problems of Prototype Theory“, in: Cognitive Linguistics. Basic Readings. Hg. v. Dirk Geeraerts. Berlin u. New York: 141-165.

Goethe, Johann Wolfgang (1999 [1787]). Die Leiden des jungen Werthers. Paralleldruck der beiden Fassungen. Studienausgabe. Hg. v. Matthias Luserke. Stuttgart.

Leroi-Gourhan, André (1980). Hand und Wort. Die Evolution von Technik, Sprache und Kunst. Frankfurt a. M.

Mellmann, Katja (2006). Emotionalisierung - Von der Nebenstundenpoesie zum Buch als Freund. Eine emotionspsychologische Analyse der Literatur der Aufklärungsepoche. Paderborn.

Mithen, Steven (1996). The Prehistory of the Mind. A Search for the Origins of Art, Religion and Science. London.

Morgan, Thomas J.H., Natalie Uomini, Luke E. Rendell et al. (2015). „Experimental evidence for the co-evolution of hominin tool-making teaching and language“, in: Nature Communications, 6. https://doi.org/10.1038/ncomms7029 (14.12.2020).

Reyer, Jürgen (2006). „Evolutionäre Bindungstheorie - Ein neuer Typ integrativer Sozialisationsforschung", in: Zeitschrift für Erziehungswissenschaft 9, Beiheft 5: 133-152.

Rosch, Elenor u. Barbara B. Lloyd (1978). Cognition and Categorization. Hillsdale (N. J.).

Seyfarth, Robert M. u. Dorothy L. Cheney (2013). „Affiliation, empathy, and the origins of theory of mind“, in: PNAS. Proceedings of the National Academy of Sciences: 10349-10356. www. pnas.org/cgi/doi/10.1073/pnas.1301223110 (14.12.2020).

Sinding, Michael (2018). „Letterier: Categories, genres, and epistolarity“, in: Was ist ein Brief? Aufsätze zu epistolarer Theorie und Kultur / What is a letter? Essays on epistolary theory and culture. Hg. v. Marie Isabel Matthews-Schlinzig u. Caroline Socha. Würzburg: 21-37.

Tooby, John u. Leda Cosmides (1992). „The Psychological Foundations of Culture“, in: The Adapted Mind. Evolutionary Psychology and the Generation of Culture. Hg. v. Jerome Barkow, Leda Cosmides u. John Tooby. New York: 19-136.

Varney, Nils R. (2002). „How Reading Works: Considerations From Prehistory to the Present“, in: Applied Neuropsychology, 9.1: 3-12. https://doi.org/10.1207/S15324826AN0901_2 (14.12.2020).

Vellusig, Robert (2020). „Literarische Anthropologie und Brief“, in: Handbuch Brief. Von der Frühen Neuzeit bis zur Gegenwart. Hg. v. Marie Isabel Matthews-Schlinzig, Jörg Schuster, Gesa Steinbrink u. Jochen Strobel. Bd. 1: Interdisziplinarität - Systematische Perspektiven Briefgenres. Berlin u. Boston: 254-268. 
Vellusig, Robert (2021). „Imagination und Inszenierung. Symbolische Distanzregulation in der Briefkultur des 18. Jahrhunderts“, in: Briefe und Tagebücher zwischen Text und Quelle. Geschichts- und Literaturwissenschaft im Gespräch II. Hg. v. Volker Depkat u. Wolfram Pyta. Berlin: 145-182.

Watzlawick, Paul, Janet H. Beavin u. Don D. Jackson (1969). Menschliche Kommunikation. Formen, Störungen, Paradoxien. Bern.

Wege, Sophia (2013). Wahrnehmung, Wiederholung, Vertikalität. Zur Theorie und Praxis der Kognitiven Literaturwissenschaft. Bielefeld. 
